\title{
CHLOROPHYTUM NEPALENSE (LINDLEY) BAKER (LILIACEAE) - A NEW ANGIOSPERMIC RECORD FOR BANGLADESH
}

\author{
Sumona Afroz ${ }^{\star}$, Ershad Tutul, Mohammad Zashim Uddin \\ and Md. Abul Hassan \\ Department of Botany, University of Dhaka, Dhaka-1000, Bangladesh
}

Key words: Chlorophytum nepalense, New record, Bangladesh

\begin{abstract}
Chlorophytum nepalense (Lindley) Baker, a perennial herb, belonging to the family Liliaceae has been described and illustrated as a new genus and species record for Bangladesh.

A perennial terrestrial herb from Runctia forest under Sherpur district of Bangladesh with white flowers from shady forest floor was collected in May 2008, and identified as Chlorophytum nepalense (Lindley) Baker by consulting the descriptions of Hooker (1892) and Xinqi and Tamura (2000).

A detailed descriptive note and illustration are prepared on the basis of a living specimen planted at the Dhaka University Botanical Garden, and also a herbarium specimen kept at the Salar Khan Herbarium, D.U.

The genus Chlorophytum Ker-Gawl., of the family Liliaceae, has not been recorded previously from the area now under Bangladesh.

Genus Chlorophytum Ker-Gawl., Bot. Mag. 27: t. 1071 (1808)

Perennial herbs, rhizomatous. Rhizome often short, inconspicuous, sometimes thick, elongate. Roots usually slightly fleshy. Leaves basal, subdistichous or fasciculate, sessile or petiolate, conduplicate. Inflorescence a terminal raceme or panicle, bract small. Scape axillary, proximately with bract-like cauline leaves. Flowers bisexual, pedicel articulate. Perianth segments 6 , usually white, free, persistent or marcescent. Stamens 6 , inserted at the base of tepals; filaments filiform, usually slightly widened near middle, anthers nearly basifixed. Ovary 3-loculed, ovules 1 to several per locule; style slender; stigma small. Fruit a capsule, acutely 3 -angled, loculicidal. Seeds black coated, flattened (Hooker 1892).
\end{abstract}

Chlorophytum nepalense (Lindley) Baker, Journ. Linn. Soc. 15: 330 (1876).

(Fig. 1)

Phalangium nepalensis Lindley, Trans. Hort. Soc. Lond. 6: 277 (1826)

Chlorophytum khasianum Hook. f., Fl. Brit. Ind. 6: 334 (1892)

Root fibrous, cylindric, nearly clustered. Rhizome often short, inconspicuous. Leaves ensiform, sessile, flat, convolute, often distichous, c $19.5 \times 4.2 \mathrm{~cm}$, acute-acuminate, linear-lanceolate, entire, margin laxly crisped, glabrous, green. Inflorescence an irregular terminal raceme, scape solid, simple or shortly branched, terete or slightly compressed, green, c $20.5 \mathrm{~cm}$ long, flowering portion c $6 \mathrm{~cm}$ long. Flowers bracteate, bracts c $0.8 \times$ $0.4 \mathrm{~cm}$, lanceolate, green, glabrous. Pedicels c $0.8 \mathrm{~cm}$ long, erect, white, jointed above the middle, perianth segments $6,3+3$, inner three c $0.8 \times 0.3 \mathrm{~cm}$, outer three $c 1.0 \times 0.2$ $\mathrm{cm}$, linear-lanceolate, acute to obtuse, white, glabrous. Stamens 6 , exserted, filaments $\mathrm{c}$ $0.3 \mathrm{~cm}$ long, white, anthers c $0.6 \mathrm{~cm}$ long, yellow, dorsifixed, dehiscence longitudinal, not recurved or revolute after flowering. Ovary c $0.25 \times$ 
*Corresponding author: E-mail: <binidu@yahoo.com>.

$0.2 \mathrm{~cm}$, green, trigonous, glabrous. Carpels 3, syncarpous, ovules many in each chamber, style

c $1.1 \mathrm{~cm}$ long, white, stigma minute, c $0.1 \mathrm{~cm}$ long, 3-fid, glabrous.

Fruit a capsule, trigonous,

c $0.8 \times 0.9 \mathrm{~cm}$, with $0.1 \mathrm{~cm} 3$-fid stigma. Seeds several per valve, c $0.3 \times 0.2 \mathrm{~cm}$, black, opaque.

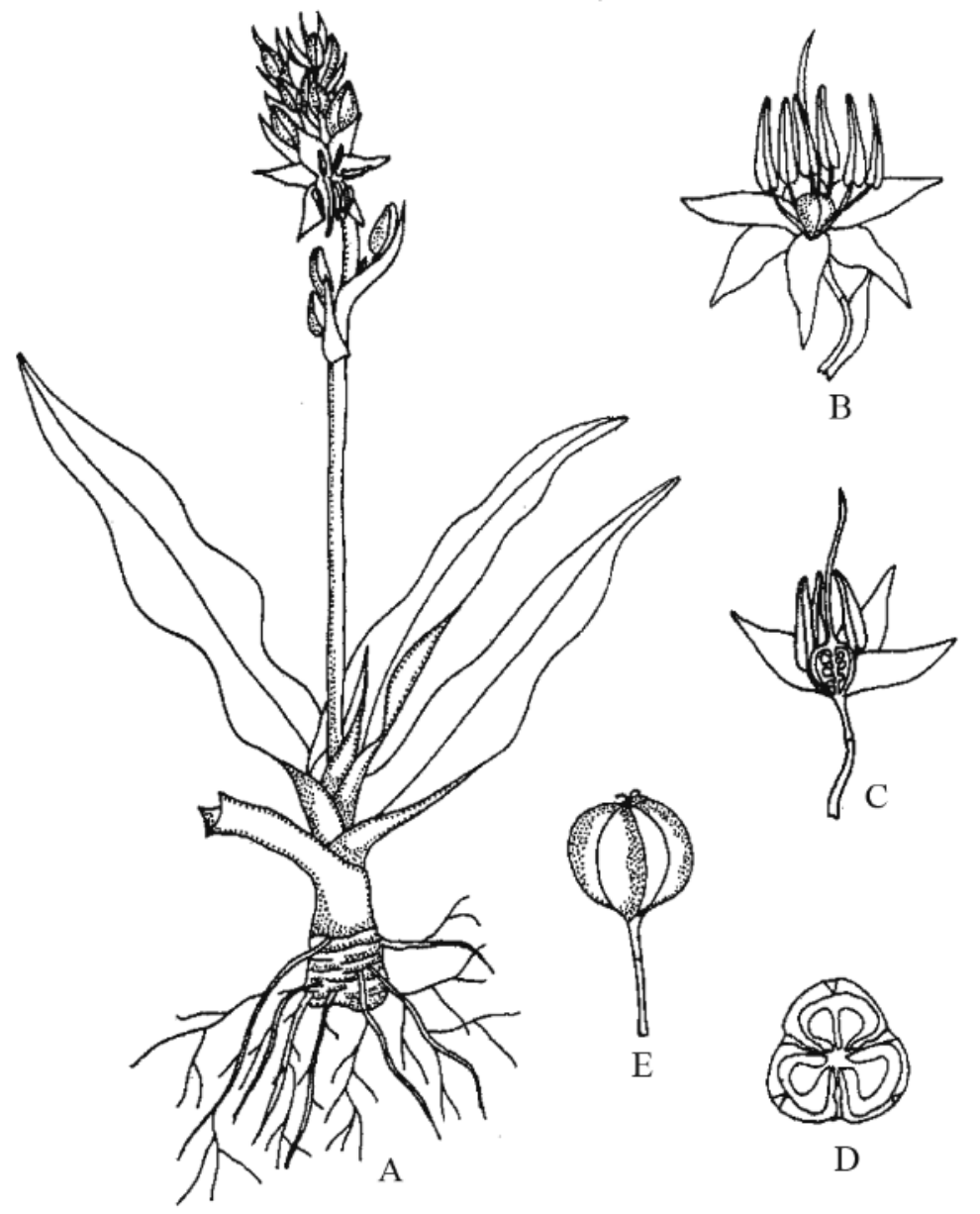

Fig. 1. Chlorophytum nepalense (Lindley) Baker, A. habit ( $\times 0.5)$; B. a flower $(\times 1)$; C. LS of a flower $(\times 1)$; D. TS of ovary $(\times 7)$; E. a fruit.

Flowering and fruiting: April to July.

Specimen examined: Sherpur: Runctia forest, 16.5.2008, Ershad Tutul, Salar Khan Herbarium, D.U. 205.

Distribution: Bangladesh, Myanmar, India, China, Nepal and Bhutan.

\section{References}

Hooker, J.D. 1892. The Flora of British India. 6: 333-335. L. Reeve \& Co. Ltd., Kent, England.

Xinqi, C. and M.N. Tamura. 2000. Chlorophytum. Flora of China, 24: 205-206. Science Press, Beizing, China. 
(Manuscript received on 8 July, 2008; revised on 20 August, 2008) 